\title{
Environmental Isolation of Circulating Vaccine-Derived Poliovirus After Interruption of Wild Poliovirus Transmission — Nigeria, 2016
}

\author{
Andrew Etsano ${ }^{1}$; Eunice Damisa, $\mathrm{MPH}^{1}$; Faisal Shuaib, $\mathrm{DrPH}^{2}$; Gatei wa Nganda ${ }^{3}$; Ogu Enemaku, PhD ${ }^{4}$; Samuel Usman, MBBS ; \\ Adekunle Adeniji, MBBS ${ }^{6}$; Jaume Jorba, $\mathrm{PhD}^{7}$; Jane Iber, MS ${ }^{7}$; Chima Ohuabunwo, $\mathrm{MD}^{3}$; Chimeremma Nnadi, MD, PhD ${ }^{3}$; Eric Wiesen, MS ${ }^{3}$
}

In September 2015, more than 1 year after reporting its last wild poliovirus (WPV) case in July 2014 (1), Nigeria was removed from the list of countries with endemic poliovirus transmission, ${ }^{*}$ leaving Afghanistan and Pakistan as the only remaining countries with endemic WPV. However, on April 29, 2016, a laboratory-confirmed, circulating vaccinederived poliovirus type 2 (cVDPV2) isolate was reported from an environmental sample collected in March from a sewage effluent site in Maiduguri Municipal Council, Borno State, a security-compromised area in northeastern Nigeria. VDPVs are genetic variants of the vaccine viruses with the potential to cause paralysis and can circulate in areas with low population immunity. The Nigeria National Polio Emergency Operations Center initiated emergency response activities, including administration of at least 2 doses of oral poliovirus vaccine (OPV) to all children aged $<5$ years through mass campaigns; retroactive searches for missed cases of acute flaccid paralysis (AFP), and enhanced environmental surveillance. Approximately 1 million children were vaccinated in the first OPV round. Thirteen previously unreported AFP cases were identified. Enhanced environmental surveillance has not resulted in detection of additional VDPV isolates. The detection of persistent circulation of VDPV2 in Borno State highlights the low population immunity, surveillance limitations, and risk for international spread of cVDPVs associated with insurgency-related insecurity. Increasing vaccination coverage with additional targeted supplemental immunization activities and reestablishment of effective routine immunization activities in newly secured and difficult-to-reach areas in Borno is urgently needed.

Borno State, located in northeast Nigeria, shares boundaries with Cameroon, Chad, and Niger (Figure 1). During the last 7 years, security challenges related to armed insurgency in the region have led to mass migration, large territories that are inaccessible to polio eradication activities, and a large population of internally displaced persons living in camps and community housing in Maiduguri, the state capital. Following the detection of the cVDPV2 isolate in Maiduguri, the Nigeria National Polio Emergency Operations Center promptly activated the

\footnotetext{
* Interruption of endemic poliovirus is likely when a country reports no new WPV case over a 1-year period. Certification of eradication occurs only when high-quality surveillance yields no evidence of WPV over a period of 3 consecutive years.
}

outbreak response standard operating procedures, which include supplemental immunization activities (SIAs) (mass campaigns intended to provide at least 2 doses of OPV to all children aged $<5$ years), retroactive searches for missed AFP cases, and enhanced environmental surveillance (2).

Genetic sequencing of the isolate indicated the virus differed by 32 nucleotides from Sabin 2 and differeed by 20 nucleotides from the closest matching sequence (VDPV2 lineage), signifying prolonged undetected circulation. This viral lineage was responsible for the 2012-2014 multicountry cVDPV2 outbreak, which started in Chad and spread to Nigeria, Niger, and Cameroon, and was last isolated in 2014 from an environmental sample collected in Yobe State, Nigeria, which borders Borno State to the west (3).

Three outbreak response SIAs were conducted in May, June, and July 2016, targeting children aged $<5$ years, and using monovalent oral poliovirus vaccine type 2 (mOPV2). The quality of the May SIA was evaluated using lot quality assurance sampling (LQAS) methodology (4). Because the cVDPV2 isolate was classified as an orphan (i.e., $>1.5 \%$ sequence difference from the nearest matching virus), indicating probable gaps in surveillance, a retroactive community AFP case search was conducted in 78,310 households and health facilities in 608 housing settlements in 10 district subdivisions (wards) bordering the environmental sample collection site.

\section{Supplemental Immunization Activities}

Among the 310 wards in Borno State's 27 local government areas (LGAs), 137 (44\%) were classified as fully accessible, and $17(6 \%)$ were accessible with military escort. The remaining 156 wards (50\%) were classified as inaccessible (Figure 2). The first of three outbreak response SIAs using mOPV2 was conducted during May 9-12, 2016, in 23 of the 27 LGAs that were fully or partially accessible in Borno State, and an additional 12 border LGAs in the adjoining Yobe, Gombe, and Adamawa states. A total of 1,329,231 children aged $<5$ years were vaccinated; LQAS results indicated that $96 \%$ of the LGAs assessed passed at a threshold of $80 \%$ coverage. The second and third SIA rounds were conducted in June and July 2016. Because of ongoing security concerns, vaccination exercises were conducted by house-to-house, border and special transit teams, and also in health camps. 
FIGURE 1. Location of the laboratory-confirmed circulating vaccine-derived poliovirus type 2 (cVDPV2) isolate reported from an environmental sewage sample - Maiduguri, Borno State, Nigeria, April 29, 2016

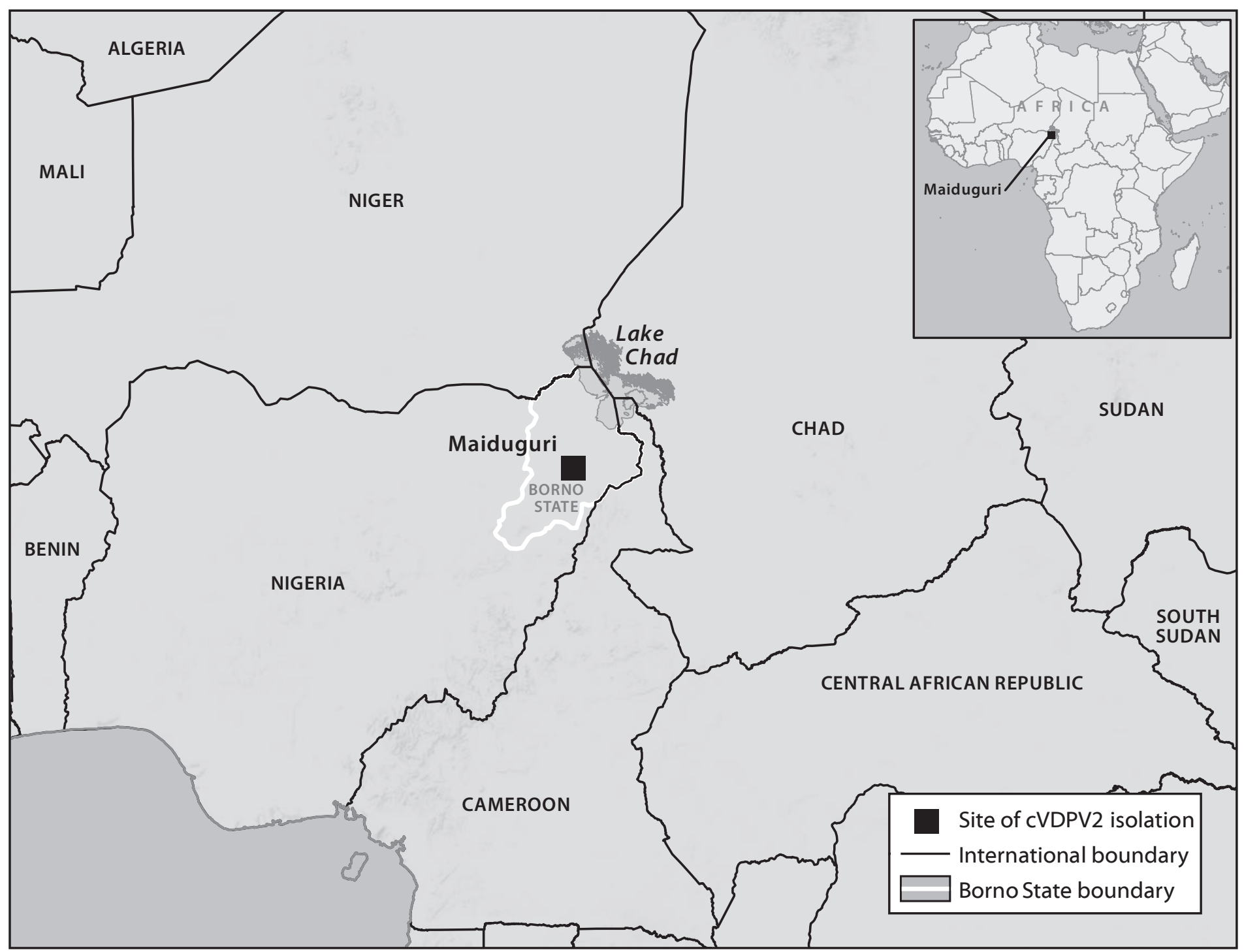

\section{Retroactive AFP Case Search}

During April 29-May 31, 2016, a total of 62 AFP cases were identified in Maiduguri Municipal Council, 13 (21\%) of which were previously unreported. For four of these 13 cases, $<60$ days had elapsed from date of onset of paralysis. Preliminary laboratory results indicate that stool samples collected from patients were negative for WPV and cVDPV; however, sample collection was conducted well beyond the recommended 14 days after paralysis onset when two stool specimens should be collected.

\section{Environmental Surveillance}

The frequency of environmental sample collection in Maiduguri was increased from monthly to weekly at each of the four environmental sampling sites. Results for samples collected through May 31, 2016, were negative for WPV and cVDPV.

\section{Discussion}

As in many countries, extensive use of OPV, a live, attenuated vaccine, contributed to the interruption of endemic WPV transmission in Nigeria. However, in settings with low routine vaccination coverage, OPV use can be associated with the emergence of VDPVs, genetic variants of the vaccine viruses with the potential to cause paralysis (5), and cVDPVs have been implicated in confirmed outbreaks (6). In April 2016, approximately 7 months after the official certification of the interruption of WPV transmission in Nigeria, environmental sampling from a sewage effluent site identified a cVDPV2 isolate in Maiduguri Municipal Council, Borno State. Genetic 


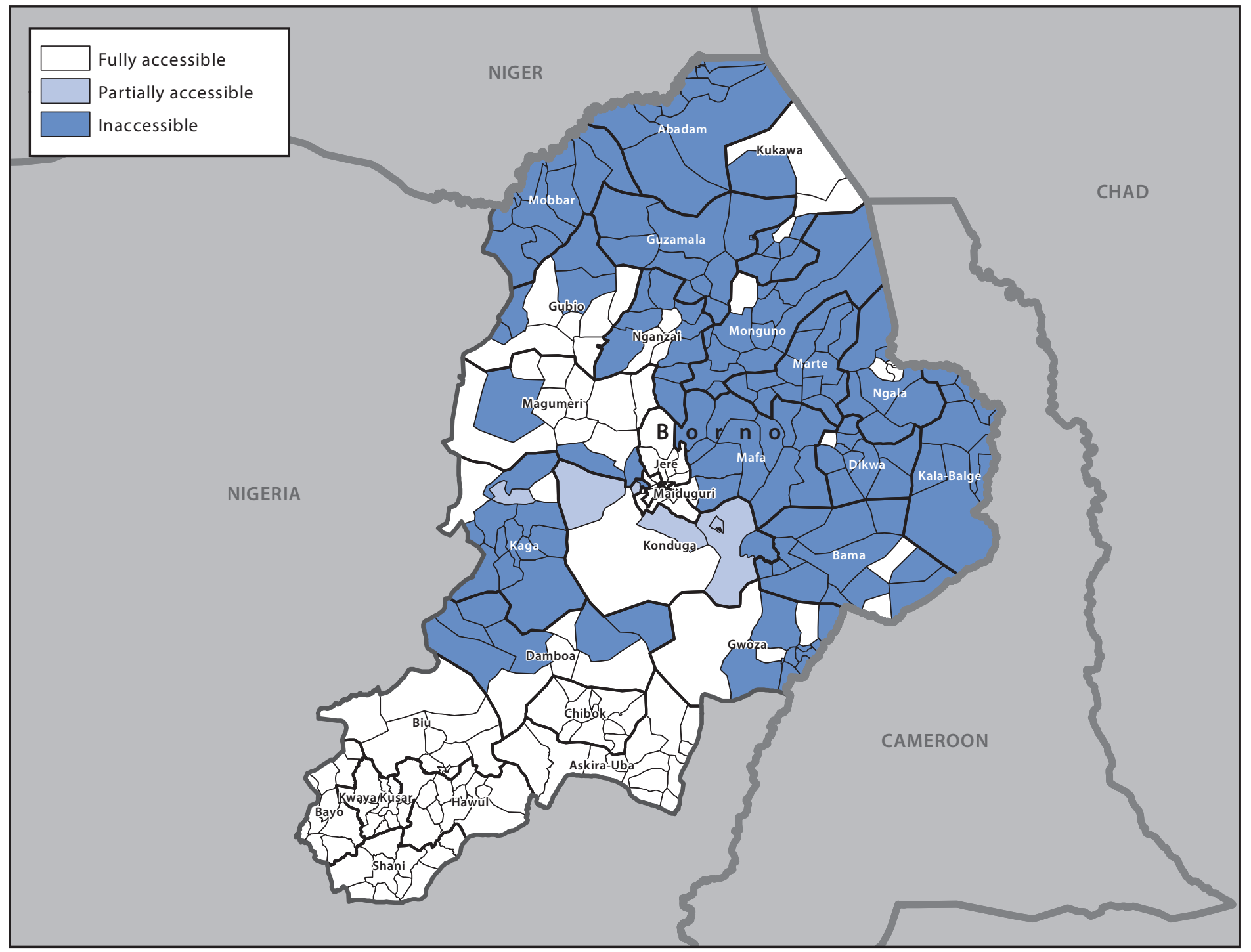

sequencing suggested that the isolated viral strain had been in circulation for at least 2 years. After activation of the Nigeria Polio Emergency Operations Center, SIAs with mOPV2 began, and a retroactive search for AFP cases identified previously unreported AFP cases.

This cVDPV2 isolate is the first to be reported in Nigeria since it was removed from the list of countries with endemic WPV transmission in September 2015, and the first to be reported from any country worldwide after the April 2016 globally coordinated switch from trivalent to bivalent OPV ( 7$)$. The primary aim of the switch was the removal from routine use of the type 2 component of OPV (OPV2), which is associated with most cVDPV outbreaks $(6,8)$. The use of mOPV2 in the response to the cVDPV2 isolation in Borno marks the first time OPV2-containing vaccine obtained from the global emergency stockpile has been deployed in an outbreak response setting, by authorization of the Director General of the World Health Organization (9).

Outbreak response to the viral isolation has been affected by difficulties in geographic access related to security challenges in the region. Evaluations conducted in Borno State during May 2016 indicated that security and accessibility remain major concerns in northeastern Nigeria. Therefore, in spite of the rapid response, immunity gaps likely exist, and the estimated number of children in the inaccessible or poorly accessible areas is currently unknown. Plans to vaccinate children in newly accessible areas and camps of internally displaced persons might mitigate, but will not eliminate, the risk for ongoing or new cVDPV2 transmission. Furthermore, poor accessibility will continue to limit high quality surveillance activities in security-compromised areas. 
Although significant progress continues to be made toward polio eradication in Nigeria, the detection of persistent cVDPV2 circulation highlights three key challenges facing Nigeria and the broader global polio eradication efforts. First, low population immunity because of inability to reach or fully immunize children in Borno State risks further outbreaks and spread of cVDPVs and other vaccine-preventable diseases (VPDs). Second, poor access to security-compromised areas might continue to limit timely detection of and appropriate response to potential outbreaks, further compounding the risks of continued disease transmission. Finally, because of large population movements across international boundaries from Borno State, the risk for international spread of cVDPVs and other VPDs remains substantially elevated in this setting.

Identification of opportunities for increasing vaccination coverage, including additional targeted SIAs and reestablishment of effective routine immunization activities in newly secured and difficult-to-reach areas in Borno, is an urgent public health need. Similar activities in neighboring state and national jurisdictions should be prioritized to limit risks for future outbreaks and spread of cVDPVs or other VPDs. A comprehensive surveillance review that aims to identify and close potential case-finding and reporting gaps, within the context of the current security situation in the area, is needed. Finally, ongoing conflict-related mass migration within and between countries in the region requires closer coordination of polio eradication activities, including SIAs and surveillance activities among affected countries, to prevent cVDPV transmission across international boundaries.

\footnotetext{
${ }^{1}$ National Primary Health Care Development Agency, Nigeria; ${ }^{2}$ Bill \& Melinda Gates Foundation, Seattle, Washington; ${ }^{3}$ Global Immunization Division, CDC; ${ }^{4}$ United Nations Children's Fund, Nigeria Office; ${ }^{5}$ CORE Group Partners Project, Nigeria; ${ }^{6}$ Division of Virology, College of Medicine, University of Ibadan, Nigeria; ${ }^{7}$ Polio And Picornavirus Laboratory Branch, Division of Viral Diseases, National Center for Immunization and Respiratory Diseases, CDC.

Corresponding author: Chimeremma Nnadi, cnnadi@cdc.gov, 404-553-7641.
}

\section{References}

1. World Health Organization. WHO removes Nigeria from polio-endemic list. Geneva, Switzerland: World Health Organization; 2016. http://www. who.int/mediacentre/news/releases/2015/nigeria-polio/en/

2. Global Polio Eradication Initiative. Standard operating procedure for responding to a poliovirus event and outbreak. Geneva, Switzerland: World Health Organization, Global Polio Eradication Initiative; 2016. http://www.polioeradication.org/Portals/0/Document/Resources/ PolioEradicators/1a.PolioOutbreakGuideline201604part2.pdf

3. Diop OM, Burns CC, Wassilak SG, Kew OM. Update on vaccine-derived polioviruses-worldwide, July 2012-December 2013. MMWR Morb Mortal Wkly Rep 2014;63:242-8.

\section{Summary \\ What is known about this topic?}

The last case of wild poliovirus transmission in Nigeria was reported in July 2014. The country was officially removed from the list of countries with endemic wild poliovirus transmission in September 2015.

What is added by this report?

In April 2016, a laboratory-confirmed isolate of circulating vaccine-derived poliovirus type 2 (cVDPV2), a genetic variant of the vaccine virus with the potential to cause paralysis, was reported from a sewage effluent site in Borno, a state in northeastern Nigeria with international boundaries. Years of armed insurgency in Borno have led to reduced polio vaccination and surveillance activities, resulting in a population of underimmunized children. The Nigeria National Polio Emergency Operations Center activated an outbreak response that included supplemental immunization activities (SIAs), a retrospective search for acute flaccid paralysis (AFP) cases, and enhanced environmental surveillance. Approximately 1 million children were vaccinated in the first SIA round, and 13 previously unreported AFP cases were identified.

What are the implications for public health practice?

Strategies for increasing vaccination coverage, including deployment of innovative approaches for reaching children in conflict-affected areas, are needed to prevent VDPV and other vaccine preventable disease (VPD) outbreaks. Strengthening surveillance is an urgent priority. Closer coordination of polio eradication activities between state and national jurisdictions in the region should be considered to prevent the potential spread of cVDPV and other VPDs across international boundaries.

4. Global Polio Eradication Initiative. Assessing vaccination coverage levels using clustered lot quality assurance sampling. Field manual. Geneva, Switzerland: World Health Organization, Global Polio Eradication Initiative; 2012. http:// www.polioeradication.org/portals/0/document/research/opvdelivery/lqas.pdf

5. Global Polio Eradication Initiative. Vaccine-derived polioviruses (VDPVs). Geneva, Switzerland: World Health Organization, Global Polio Eradication Initiative; 2010. http://www.polioeradication.org/polioandprevention/thevirus/ vaccinederivedpolioviruses.aspx

6. Global Polio Eradication Initiative. Fact sheet: vaccine-derived poliovirus. Geneva, Switzerland: World Health Organization, Global Polio Eradication Initiative; 2015. http://www.polioeradication.org/Portals/0/ Document/Polioandprevention/CVDPVFactSheetMarch2015.pdf

7. Morales M, Nnadi CD, Tangermann RH, Wassilak SG. Notes from the field: circulating vaccine-derived poliovirus outbreaks - five countries, 2014-2015. MMWR Morb Mortal Wkly Rep 2016;65:128-9. http://dx.doi.org/10.15585/ mmwr.mm6505a5

8. World Health Organization. Replacing trivalent OPV with bivalent OPV: a critical step in polio eradication. http://www.who.int/immunization/ diseases/poliomyelitis/endgame_objective2/oral_polio_vaccine/en/

9. Global Polio Eradication Initiative. Operational framework for monovalent oral poliovirus type 2 (mOPV2) deployment and replenishment. Geneva, Switzerland: World Health Organization, Global Polio Eradication Initiative; 2015. http://www.polioeradication.org/Portals/0/Document/ Resources/PostEradication/mOPV2_Operational_Framework.pdf 